\title{
An Improved Cross Talk Cancelling Digital Predistortion for MIMO Transmitters
}

\author{
Zhiliang Zhang, ${ }^{1,2}$ Ying Shen, ${ }^{1}$ Shihai Shao, ${ }^{1}$ Wensheng Pan, ${ }^{1}$ and Youxi Tang ${ }^{1}$ \\ ${ }^{1}$ National Key Laboratory of Communications, University of Electronic Science and Technology of China, \\ Chengdu, Sichuan 611731, China \\ ${ }^{2}$ Department of Electronic Information Engineering, Jincheng College, Sichuan University, Chengdu, Sichuan 611731, China \\ Correspondence should be addressed to Ying Shen; shenying@uestc.edu.cn
}

Received 8 December 2015; Revised 25 February 2016; Accepted 3 March 2016

Academic Editor: Yuh-Shyan Chen

Copyright (C) 2016 Zhiliang Zhang et al. This is an open access article distributed under the Creative Commons Attribution License, which permits unrestricted use, distribution, and reproduction in any medium, provided the original work is properly cited.

\begin{abstract}
An improved cross talk cancelling digital predistortion (ICTC-DPD) with novel estimating technique and decoupling technique is proposed, which can be used to compensate the cross talk with possible delay and compensate the nonlinearity with memory effect in MIMO transmitting channels. The cross talk cancellation ability, which is defined as the cross talk power ratio before cancellation to after cancellation in decibel, is analyzed and its closed-form expression is derived. Simulation results prove that the ICTC-DPD can achieve higher cross talk estimation accuracy and higher cross talk cancellation ability than the original CTC-DPD. Experimental results show that the ICTC-DPD can achieve $20 \mathrm{~dB}$ cross talk cancellation for two channels transmitting $20 \mathrm{MHz}$ LTE signals.
\end{abstract}

\section{Introduction}

Multiple-input multiple-output (MIMO) technology is one of the most promising solutions in improving the spectral efficiency of wireless systems [1-5] and has been used in several wireless communication standards, such as LTE and LTEAdvanced [6]. In the MIMO transmitter design, nonlinearity is a basic issue [7-13]. It is mainly introduced by the inherently nonlinear power amplifiers (PAs) and by the cross talk (coupling) which is occurring before PAs and distorted by PAs. It will degrade the quality of the output signals and thus degrade the performance of the MIMO communication system [7]. It is worth pointing out that the cross talk also occurs at the circuit after PAs and at the antennas; however, when the preceding nonlinearity is compensated, this cross talk can be compensated at the receiver side concurrently with the composite cross talk generated at the receiver antennas and by the channel $[14,15]$.

In some proposed techniques in open literatures, the PAs' nonlinearity and the distorted cross talk have been compensated concurrently. These techniques can be divided into two kinds. The first is crossover DPD (CO-DPD) $[7,8]$. In CO$\mathrm{DPD}$, each direct path or coupling path uses a predistorter of memory polynomial model to compensate, where the possible delay of cross talk can be considered as memory effect and thus can be compensated. However, it ignores the higherorder cross-terms, which results in a loss of performance, especially at high cross talk levels [9], and it has high complexity because too many predistorters are used. The second is cross talk cancelling DPD (CTC-DPD) [9-11], where an inversion of coupling matrix is introduced after predistorters for decoupling to make the channels between predistorters and PAs independent, and therefore only $M$ predistorters of memory polynomial model are needed by $M$ outputs. However, for CTC-DPD, the coupling matrix estimated in the first round will be distorted by the nonlinearity of PAs, and the propagation of this error will decrease the accuracy of the final estimated values at the second round. Moreover, its estimating technique needs the output of all channels simultaneously, which makes shared feedback impossible. Additionally, the inversion of coupling matrix needs to be computed for decoupling, which results in an increase of complexity. Furthermore, the delay of cross talk is ignored, which leads to a loss of performance in a particular situation where the two adjacent channels have significant interval and transmit rapid baseband signal at high power. 


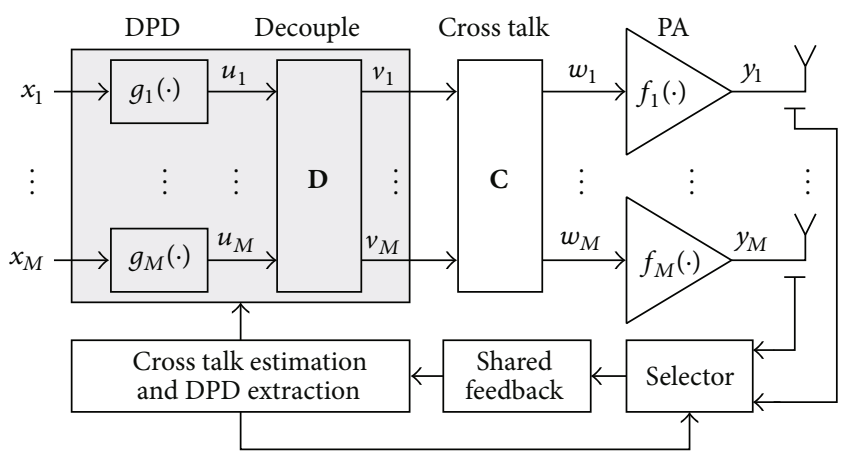

Figure 1: The system model of an $M$-output MIMO transmitter with ICTC-DPD, where the cross talk and nonlinearity can be compensated by shared feedback.

In this paper, an improved CTC-DPD (ICTC-DPD) with novel estimating and decoupling technique based on shared feedback is proposed. The system model of ICTC-DPD is shown in Figure 1. In ICTC-DPD, the novel estimating technique can estimate the cross talk with delay avoiding the distortion of PA nonlinearity, while the novel decoupling technique has low complexity and can compensate the cross talk with delay. By using shared feedback, inconsistency of output channels can be compensated too.

\section{System Model}

In this section, we will first review the original CTC-DPD and then give out our improvement on estimating technique and decoupling technique.

2.1. Original CTC-DPD. The original CTC-DPD is proposed and analyzed for a 2-output MIMO. Its system model is similar to Figure 1 but with individual feedback [9-11], and its process can be described by

$$
\begin{aligned}
& \mathbf{w}=\mathbf{u D C}=\mathbf{u I}=\mathbf{u}, \\
& \mathbf{y}_{i}=f_{i}\left(\mathbf{w}_{i}\right)=f_{i}\left(\mathbf{u}_{i}\right)=f_{i}\left(g_{i}\left(\mathbf{x}_{i}\right)\right)=\mathbf{x}_{i},
\end{aligned}
$$

where $\mathbf{u}=\left[\begin{array}{ll}\mathbf{u}_{1} & \mathbf{u}_{2}\end{array}\right], \mathbf{v}=\left[\begin{array}{ll}\mathbf{v}_{1} & \mathbf{v}_{2}\end{array}\right]$, and $\mathbf{w}=\left[\begin{array}{ll}\mathbf{w}_{1} & \mathbf{w}_{2}\end{array}\right]$ are the equivalent baseband signals before decoupling (after predistortion), after decoupling (before coupling), and after coupling (the input of the PAs), $\mathbf{x}_{i}$ is the baseband input, and $\mathbf{y}_{i}$ is the equivalent baseband signal of PA output of the $i$ th channel, respectively.

$\mathbf{C}=\left[\begin{array}{cc}1 & C_{12} \\ C_{21} & 1\end{array}\right]$ is the coupling matrix describing the cross talk before the PAs, where $C_{i j}$ is the coupling coefficient from the $i$ th channel to the $j$ th channel and $C_{11}=C_{22}=1,0<$ $\left\{\left|C_{12}\right|,\left|C_{21}\right|\right\}<1$. It is easy to know that $\mathbf{C}$ is invertible.

$\mathbf{D}$ is the decoupling matrix, and it is the inversion of $\mathbf{C}$; that is, $\mathbf{D}=\mathbf{C}^{-1}=\left(1 /\left(1-C_{12} C_{21}\right)\right)\left[\begin{array}{cc}1 & -C_{12} \\ -C_{21} & 1\end{array}\right]$. As can be seen in (1), with the decoupling, the cross talk before the PAs is compensated and the channels before the PAs are independent.
The predistortion function $g_{i}(\cdot)$ is used to compensate the nonlinear function $f_{i}(\cdot)$ of the $i$ th PA, which is described by odd-order-only memory polynomial model [16] as follows:

$$
\begin{aligned}
y_{i}(n) & =f_{i}\left(w_{i}(n)\right) \\
& =\sum_{q=0}^{Q} \sum_{\substack{k=1 \\
k \text { odd }}}^{K} h_{k, q}^{(i)}\left|w_{i}(n-q)\right|^{k-1} w_{i}(n-q),
\end{aligned}
$$

where $h_{k, q}^{(i)}$ is the $k$-order polynomial coefficient of the $q$ th filter tap and $K$ and $Q$ are the maximum polynomial order and memory depth, respectively. For a block of samples, (3) can be expressed in a matrix form as

$$
\mathbf{y}_{i}=\mathbf{A}_{\mathbf{w}_{i}} \mathbf{h}_{i},
$$

where $\mathbf{w}_{i}=\left[\begin{array}{llll}w_{i}(1) & w_{i}(2) & \cdots & w_{i}(N)\end{array}\right]^{T}$ and $\mathbf{y}_{i}=$ $\left[\begin{array}{llll}y_{i}(1) & y_{i}(2) & \cdots & y_{i}(N)\end{array}\right]^{T}$ are the vectors of $N$ samples of complex envelope of input and output signal and $\mathbf{h}_{i}=$ $\left[\begin{array}{llllllll}h_{1,0}^{(i)} & h_{3,0}^{(i)} & \cdots & h_{K, 0}^{(i)} & \cdots & h_{1, \mathrm{Q}}^{(i)} & h_{3, \mathrm{Q}}^{(i)} & h_{K, \mathrm{Q}}^{(i)}\end{array}\right]^{T}$ is the vector of the memory polynomial coefficients, respectively. Consider $\mathbf{A}_{\mathbf{w}_{i}}=\left[\begin{array}{llllll}\mathbf{a}_{\mathbf{w}_{i}}^{0} & \mathbf{a}_{\mathbf{w}_{i}}^{1} & \cdots & \mathbf{a}_{\mathbf{w}_{i}}^{q} & \cdots & \mathbf{a}_{\mathbf{w}_{i}}^{Q}\end{array}\right]$, and

$$
=\left[\begin{array}{cccc}
\mathbf{a}_{\mathbf{w}_{i}}^{q} \\
=\left[\begin{array}{cccc}
\mathbf{0}_{q \times 1} & \mathbf{0}_{q \times 1} & \cdots & \mathbf{0}_{q \times 1} \\
a_{1}\left(w_{i}(1)\right) & a_{2}\left(w_{i}(1)\right) & \cdots & a_{K}\left(w_{i}(1)\right) \\
\vdots & \vdots & \cdots & \vdots \\
a_{1}\left(w_{i}(N-q)\right) & a_{2}\left(w_{i}(N-q)\right) & \cdots & a_{K}\left(w_{i}(N-q)\right)
\end{array}\right],
\end{array}\right.
$$

where $a_{k}\left(w_{i}(n)\right)=\left|w_{i}(n)\right|^{k-1} w_{i}(n)$ and $\mathbf{0}_{q \times 1}=\underbrace{\left[\begin{array}{llll}0 & 0 & \cdots & 0\end{array}\right]^{T}}_{q \text { zeros }}$.

In this technique, the coupling matrix and DPD coefficients are estimated by two rounds. In the first round, the coupling matrix is estimated by

$$
\widehat{\mathbf{C}}=\operatorname{pinv}(\mathbf{v}) \mathbf{y},
$$

where $\operatorname{pinv}(\cdot)$ is the pseudoinverse function and the DPD coefficients are extracted by

$$
\left[\begin{array}{ll}
\mathbf{d}_{1} & \phi_{1} \\
\boldsymbol{\phi}_{2} & \mathbf{d}_{2}
\end{array}\right]=\operatorname{pinv}\left(\left[\begin{array}{ll}
\mathbf{A}_{\mathbf{y}_{1}} & \mathbf{A}_{\mathbf{y}_{2}}
\end{array}\right]\right)\left[\begin{array}{ll}
\mathbf{v}_{1} & \mathbf{v}_{2}
\end{array}\right] \widehat{\mathbf{C}}
$$

where $\mathbf{d}_{i}=\left[\begin{array}{llllllll}d_{1,0}^{(i)} & d_{3,0}^{(i)} & \cdots & d_{K, 0}^{(i)} & \cdots & d_{1, Q}^{(i)} & d_{3, Q}^{(i)} & d_{K, Q}^{(i)}\end{array}\right]^{T}$ is the vector of memory polynomial coefficients of the $i$ th predistorter. In the second round, a more accurate coupling matrix is estimated by

$$
\widehat{\mathbf{C}}=\operatorname{pinv}\left(\left[\begin{array}{ll}
\mathbf{v}_{1} & \mathbf{v}_{2}
\end{array}\right]\right)\left[\begin{array}{ll}
\mathbf{A}_{\mathbf{y}_{1}} \mathbf{d}_{1} & \mathbf{A}_{\mathbf{y}_{2}} \mathbf{d}_{2}
\end{array}\right]
$$

and more accurate DPD coefficients are extracted using (7) with $\widehat{\mathbf{C}}$ from (8). However, first, the coupling matrix estimated in the first round will be distorted by the nonlinearity of PAs, and the propagation of this error will reduce the accuracy of the final estimated values. Second, this estimating technique needs the output of all channels simultaneously, which makes shared feedback impossible. 
2.2. The Proposed Estimating Technique. The cross talk usually takes place between two adjacent channels with little interval; therefore the delay of its baseband signal is significantly small and is usually ignored, while the phase delay of the carrier is combined into the coupling coefficient. However, in a particular situation, such as that where the two adjacent channels have significant interval and transmit rapid baseband signal at high power, the baseband of cross talk may have a noticeable delay. Here we propose a novel cross talk estimating technique which can estimate both the coupling coefficient and the baseband delay of cross talk by shared feedback and improve the DPD extraction to adapt that.

When $C_{i j}$ and $d_{i j}$ (the corresponding baseband delay) are being estimated, only a sequence of MPSK symbols $v_{i}^{\prime}(n)$ and the corresponding sequence of $-\widetilde{C}_{i j} v_{i}^{\prime}\left(n-\widetilde{d}_{i j}\right.$ ) (where gain coefficient $\widetilde{C}_{i j}$ and delay $\widetilde{d}_{i j}$ are trial values) are sent to the $i$ th and the $j$ th digital-to-analog converters (DACs), respectively. The other channels keep silent. The input of the $j$ th PA with noise is

$$
w_{j}^{\prime}(n)=C_{i j} v_{i}^{\prime}\left(n-d_{i j}\right)-\widetilde{C}_{i j} v_{i}^{\prime}\left(n-\widetilde{d}_{i j}\right)+n_{j}(n) .
$$

Assume $n_{j}(n)$ is statistical independent noise with power spectral density $N_{0}$. The power of $w_{j}^{\prime}(n)$ is

$$
\begin{aligned}
E\left\{\left|w_{j}^{\prime}(n)\right|^{2}\right\}= & 0.5\left(\left|C_{i j}\right|^{2}+\left|\widetilde{C}_{i j}\right|^{2}\right) P_{i} \\
& -\operatorname{Re}\left\{C_{i j}^{*} \widetilde{C}_{i j} R_{v i}\left(d_{i j}-\widetilde{d}_{i j}\right)\right\}+B N_{0},
\end{aligned}
$$

where $B$ is the bandwidth and $R_{v i}(\cdot)$ and $P_{i}$ are the autocorrelation function and power of $v_{i}^{\prime}(n)$, respectively.

For given $\widetilde{d}_{i j}$, the gradient descent method [17] is used to search $\widetilde{C}_{i j}$ which minimizes the power of $w_{j}^{\prime}(n)$. It can be proved that this problem is convex, and $w_{j}^{\prime}(n)$ gets minimal power $0.5\left|C_{i j}\right|^{2} P_{i}\left(1-\left|R_{v i}\left(d_{i j}-\widetilde{d}_{i j}\right) / P_{i}\right|^{2}\right)+B N_{0}$ when $\widetilde{C}_{i j}=\left(R_{v i}^{*}\left(d_{i j}-\widetilde{d}_{i j}\right) / P_{i}\right) C_{i j}$. Based on the characteristic of autocorrelation function, it is known that $w_{j}^{\prime}(n)$ gets final minimal power $B N_{0}$ when $\widetilde{d}_{i j}=d_{i j}$ and $\widetilde{C}_{i j}=C_{i j}$. Therefore, we can use the preknowledge of channel interval to get approximate $\dot{d}_{i j}$ and then vary $\tilde{d}_{i j}$ about $\dot{d}_{i j}$ and repeat the search to let $w_{j}^{\prime}(n)$ get final minimal power, where $\widetilde{d}_{i j}$ and $\widetilde{C}_{i j}$ are as the estimated values and can be denoted as

$$
\left(\widehat{C}_{i j}, \widehat{d}_{i j}\right)=\underset{\tilde{C}_{i j} \in \mathbb{C}, \tilde{d}_{i j} \in \mathbb{Z}}{\arg \min } E\left\{\left|w_{j}^{\prime}(n)\right|^{2}\right\} .
$$

Due to practical limitations, it may not be feasible to add extra RF hardware to receive $w_{j}^{\prime}(n)$, so the parameters can be estimated using the output of the PA, that is, $y_{j}^{\prime}(n)$. Its power can be attained by calculating the average energy of $N$ samples. Therefore, the parameters can be estimated by

$$
\left(\widehat{C}_{i j}, \widehat{d}_{i j}\right)=\underset{\widetilde{C}_{i j} \in \mathbb{C}, \tilde{d}_{i j} \in \mathbb{Z}}{\arg \min }\left\{\frac{1}{N} \sum_{j=1}^{N}\left|y_{j}^{\prime}(j)\right|^{2}\right\} .
$$

Although the PA has nonlinearity, the estimation in (12) will not be distorted by the PA's nonlinearity. There are two reasons. First, MPSK symbols with fixed magnitude are chosen as stimulus to make (9), that is, the input of the PA, has fixed magnitude. In this situation, the memory polynomial model in (3) can be simplified as $y_{i}(n)=\sum_{q=0}^{\mathrm{Q}} h_{q}^{(i)} w_{i}(n-q)$, where the nonlinearity is avoided and only the memory effect is left. Second, since PA behaves linearly at enough backoff [16], based on (10), we can let the target PA work at linear region by choosing small magnitude MPSK symbols and small initial $\widetilde{C}_{i j}$ in the gradient descent method.

When all cross talk has been estimated, the $j$ th predistorter memory polynomial coefficients can be extracted by

$$
\mathbf{d}_{j}=\operatorname{pinv}\left(\mathbf{A}_{\mathbf{y}_{j}}\right)\left[\mathbf{v}_{j}+\sum_{i=1, i \neq j}^{M} \widehat{C}_{i j} \delta\left(n-\widehat{d}_{i j}\right) * \mathbf{v}_{i}\right],
$$

where $\delta\left(n-\widehat{d}_{i j}\right) * \mathbf{v}_{i}$ means that the vector of $\mathbf{v}_{i}$ should delay $\widehat{d}_{i j}$ samples. Because the different channels are received by shared feedback, the channel inconsistency can be compensated too.

2.3. The Proposed Decoupling Technique. Assume the transmitting channels are placed sequentially. Because the cross talk between nonadjacent channels is usually significantly small and thus can be ignored, the estimated coupling matrix of $M$ transmitting channels can be denoted as

$$
\widehat{\mathbf{C}}=\left[\begin{array}{ccccc}
1 & \widehat{C}_{12} & & & 0 \\
\widehat{C}_{21} & 1 & \widehat{C}_{23} & & \\
& \cdots & \cdots & \cdots & \\
& & \widehat{C}_{(M-1)(M-2)} & 1 & \widehat{C}_{(M-1) M} \\
0 & & & \widehat{C}_{M(M-1)} & 1
\end{array}\right]
$$

For large $M$, it is a sparse matrix.

The original CTC-DPD uses the inversion of $\widehat{\mathbf{C}}$, which is no longer a sparse matrix, as decoupling matrix. However, the computation of the inversion and the implement of dense decoupling matrix multiplication will increase the complexity of the technique, especially with large $M$. Further, the baseband delay of cross talk, if it exists in the particular situation, cannot be compensated by this technique.

In order to compensate the cross talk with possible delay simply, here we use

$$
D_{i j}= \begin{cases}1 & i=j \\ -\widehat{C}_{i j} \delta\left(n-\widehat{d}_{i j}\right) * & i \neq j\end{cases}
$$

for decoupling, where $\delta\left(n-\widehat{d}_{i j}\right) *$ means that the vector of corresponding input should delay $\widehat{d}_{i j}$ samples to take part in decoupling. When $\widehat{\mathbf{C}}$ is sparse, this decoupling matrix is sparse too. 
With our decoupling technique, the equivalent baseband signal of the $i$ th PA input is

$$
\begin{aligned}
w_{i}(n)= & u_{i}(n) \\
& +\sum_{j=1, j \neq i}^{M}\left[C_{j i} u_{j}\left(n-d_{j i}\right)-\widehat{C}_{j i} u_{j}\left(n-\widehat{d}_{j i}\right)\right] \\
& -\sum_{j=1, j \neq i}^{M} \widehat{C}_{i j} C_{j i} u_{i}\left(n-\widehat{d}_{i j}-d_{j i}\right) \\
& -\sum_{j=1, j \neq i}^{M} \sum_{k=1, k \neq j, k \neq i}^{M} \widehat{C}_{k j} C_{j i} u_{k}\left(n-\widehat{d}_{k j}-d_{j i}\right) .
\end{aligned}
$$

When the non-adjacent-channel cross talk and the delay of cross talk baseband are ignored, (16) can be rewritten as

$$
\begin{aligned}
w_{i}(n)= & {\left[1-\widehat{C}_{i(i-1)} C_{(i-1) i}-\widehat{C}_{i(i+1)} C_{(i+1) i}\right] u_{i}(n) } \\
& +\left(C_{(i-1) i}-\widehat{C}_{(i-1) i}\right) u_{i-1}(n) \\
& +\left(C_{(i+1) i}-\widehat{C}_{(i+1) i}\right) u_{i+1}(n) \\
& -\widehat{C}_{(i-2)(i-1)} C_{(i-1) i} u_{i-2}(n) \\
& -\widehat{C}_{(i+2)(i+1)} C_{(i+1) i} u_{i+2}(n) .
\end{aligned}
$$

In general, the coupling coefficient is significantly small. Therefore, the terms with second-order coupling coefficient in (16) and (17), which represent the recoupled decoupling components, can be ignored. Further, when there is no estimation error, (16) and (17) can be approximated as $w_{i}(n) \approx$ $u_{i}(n)$, where the cross talk is cancelled.

\section{Analysis of Cross Talk Cancellation Ability}

In this section, we investigate the impact of the estimation errors of coupling coefficient and time delay on the cross talk cancellation ability, which is defined as the cross talk power ratio before cancellation to after cancellation in decibel. Because the time delay may not be integer multiple of sampling time, the analysis is done in continuous time domain.

The cancellation ability of the cross talk from the $i$ th channel to the $j$ th channel is given by

$$
\Psi_{i j}=10 \log \frac{P_{i j}^{\circ}}{P_{i j}}=-10 \log \frac{E\left\{\left|c_{i j}(t)\right|^{2}\right\}}{E\left\{\left|c_{i j}^{\circ}(t)\right|^{2}\right\}},
$$

where $P_{i j}^{\circ}$ and $P_{i j}$ denote the power of the cross talk from the $i$ th channel to the $j$ th channel before cancellation and after cancellation,

$$
c_{i j}^{\circ}(t)=C_{i j} u_{i}\left(t-\tau_{i j}\right)
$$

denotes the baseband of original cross talk before cancellation ( $\tau_{i j}$ is the time delay), and

$$
\begin{aligned}
c_{i j}(t)= & C_{i j} u_{i}\left(t-\tau_{i j}\right)-\widehat{C}_{i j} u_{i}\left(t-\widehat{\tau}_{i j}\right) \\
& -\sum_{k=1, k \neq j, k \neq i}^{M} \widehat{C}_{i k} C_{k j} u_{i}\left(t-\widehat{\tau}_{i k}-\tau_{k j}\right)
\end{aligned}
$$

denotes the baseband of residual cross talk after cancellation ( $\widehat{\tau}_{i j}$ is the estimation of time delay), respectively.

Because the coupling coefficient is significantly small, the last term in (20), which represents the cancellation error items introduced by the other channels, is much weaker than the first two terms; therefore we can approximate (20) as

$$
c_{i j}(t) \approx C_{i j} u_{i}\left(t-\tau_{i j}\right)-\widehat{C}_{i j} u_{i}\left(t-\widehat{\tau}_{i j}\right) .
$$

In practice, the coupling coefficient and time delay are subject to certain estimation errors. Here, we build an error model as follows:

$$
\begin{aligned}
\widehat{C}_{i j} & =(1+\eta) e^{j \varphi} C_{i j}, \\
\widehat{\tau}_{i j} & =\tau_{i j}+\mu,
\end{aligned}
$$

where $\eta, \varphi$, and $\mu$ are coupling coefficient magnitude error, coupling coefficient phase error, and time delay error, respectively.

With (18)-(22), we can attain

$$
\begin{aligned}
\Psi_{i j} & \approx-10 \log \left[2+2 \eta+\eta^{2}\right. \\
- & \left.\frac{2(1+\eta) \operatorname{Re}\left\{R_{i}(\mu) e^{-j \varphi}\right\}}{R_{i}(0)}\right],
\end{aligned}
$$

where $R_{i}(\cdot)$ is the autocorrelation function of the $i$ th channel baseband signal $u_{i}(t)$. The proof can be seen in Appendix.

\section{Results and Discussions}

In this section, simulation and experimental results are presented to evaluate the performance of our estimating and decoupling technique.

Figures 2 and 3 show the simulation results of magnitude and phase relative error of estimated coupling coefficient versus signal-to-noise ratio (SNR) of feedback in a 2-output MIMO, where the cross talk is set as $-15 \mathrm{~dB}$ with phase delay $0.6 \pi$. Because of the inherent thermal noise and analog-todigital converter (ADC) quantizing noise on the feedback, here feedback SNR is considered. In the two figures, the distorted results, which are attained by (6) and thus distorted by the nonlinearity of PAs, have the highest relative error, the CTC-DPD results, which are attained by the CTC-DPD and suffering error propagation, have the middle relative error, and the ICTC-DPD results, which avoid the distortion of PAs, have the lowest relative error. The relative error of these techniques does not monotonously decrease when feedback SNR 


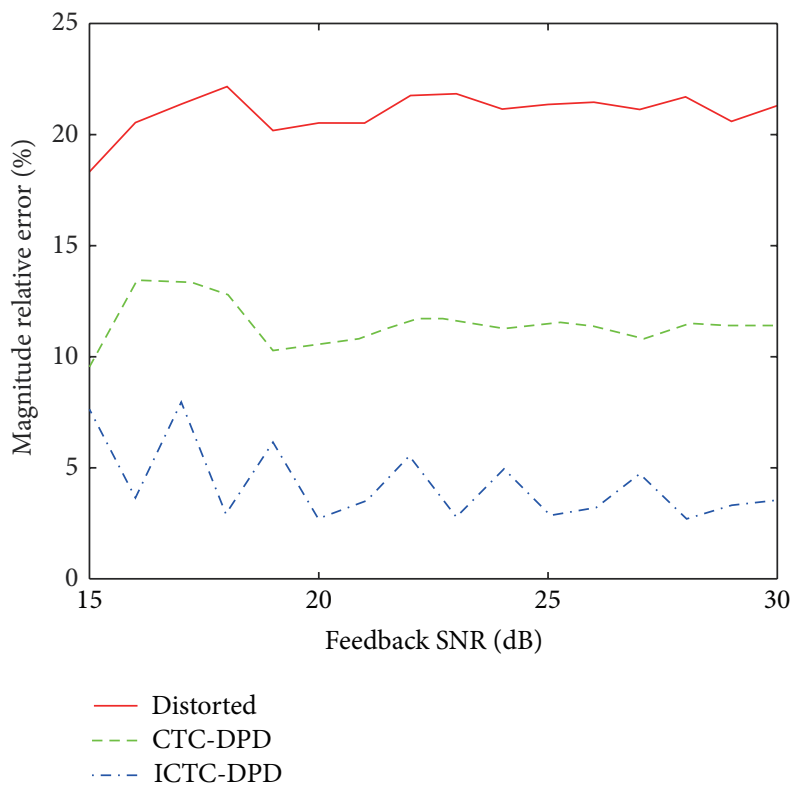

FIGURE 2: Simulation results of magnitude relative error of coupling coefficient estimated by different techniques in a 2-output MIMO.

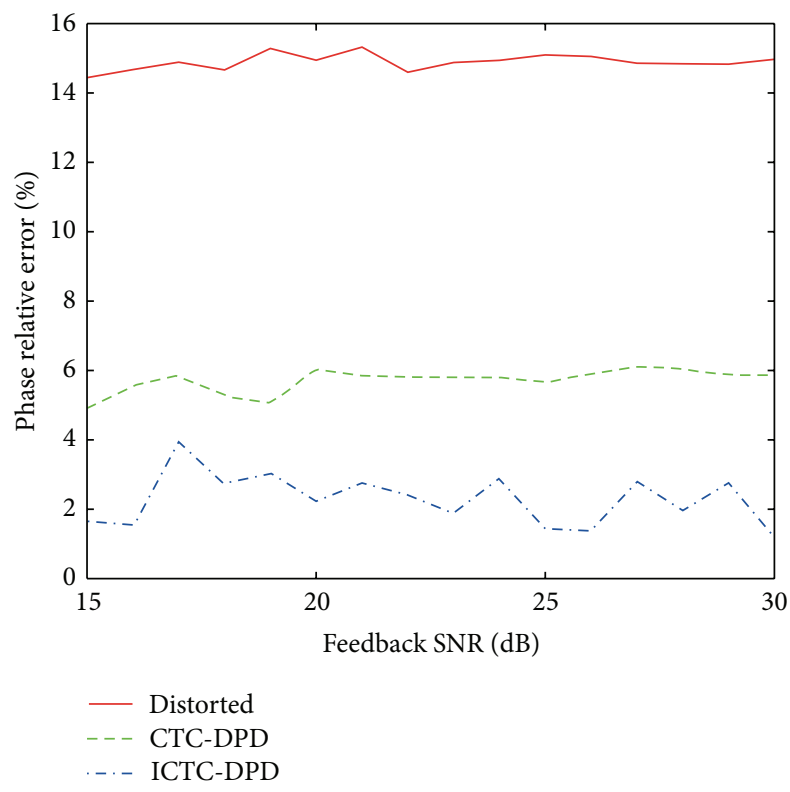

FIGURE 3: Simulation results of phase relative error of coupling coefficient estimated by different techniques in a 2-output MIMO.

increases, because of the computing precision problem when the feedback SNR is significantly high and the magnitude or phase of coupling coefficient is significantly small.

Figures 4-7 show the simulation cancellation ability of the cross talk between adjacent transmitting channels. The simulation conditions are as follows: carrier frequency is $2.45 \mathrm{GHz}$; 16 QAM is used with symbol rate $10 \mathrm{M} / \mathrm{s}$; root raised cosine pulse with 0.22 roll-off factor is used for pulse shaping. The analytical results are produced directly by (23), and the simulation results are calculated according to (18).

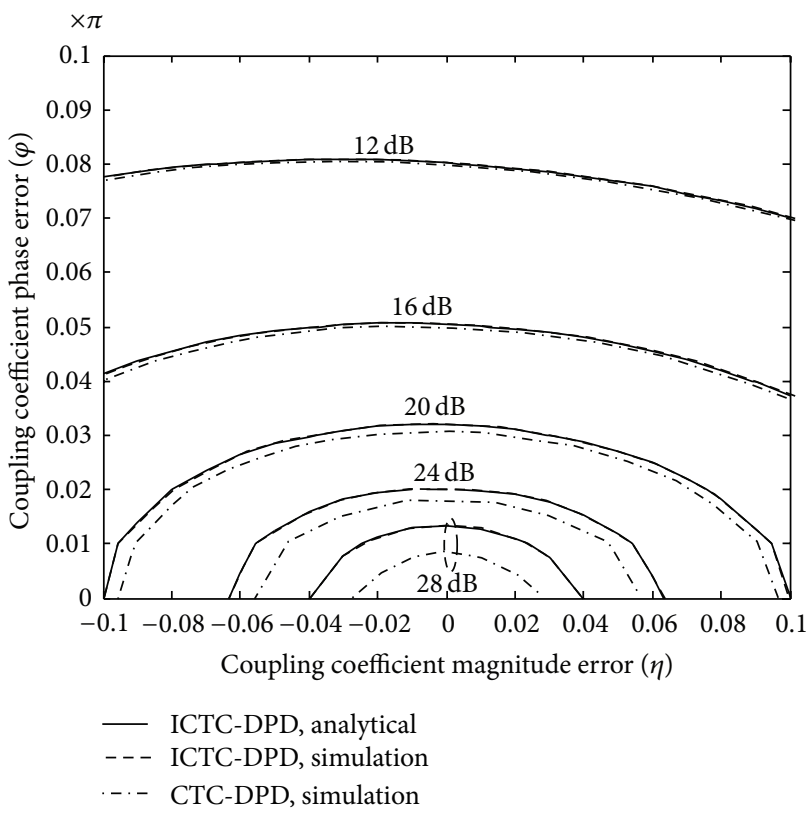

FIGURE 4: Simulation results of cross talk cancellation ability contour in a 2-output MIMO, where the cross talk delay of $0.4 \mathrm{~ns}$ is compensated by the ICTC-DPD. It shows that the ICTC-DPD can achieve higher cross talk cancellation ability than CTC-DPD when the same phase error and magnitude error of coupling coefficient estimationexist.

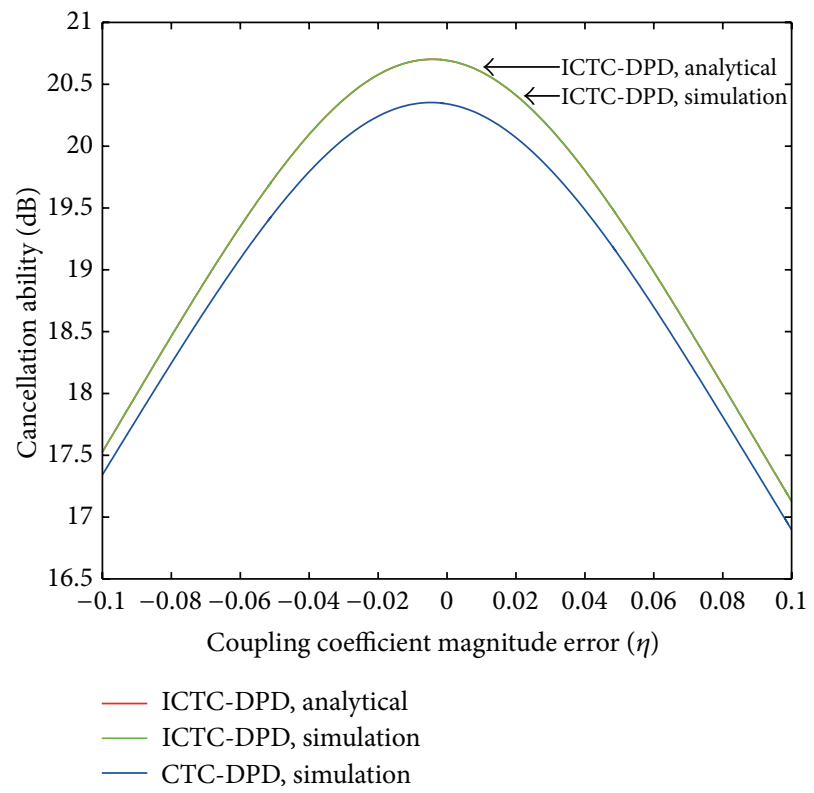

FIGURE 5: Cross talk cancellation ability varies coupling coefficient magnitude error when phase error $\varphi=0.03 \pi$ in a 2-output MIMO.

The results shown in Figures 4-6 are of a 2-output MIMO, where the cross talk delay of $0.4 \mathrm{~ns}$ (which relates to about $12 \mathrm{~cm}$ channel interval) is compensated by the ICTC-DPD. Among them, Figure 4 gives the cross talk cancellation ability changing with coupling coefficient magnitude error and 


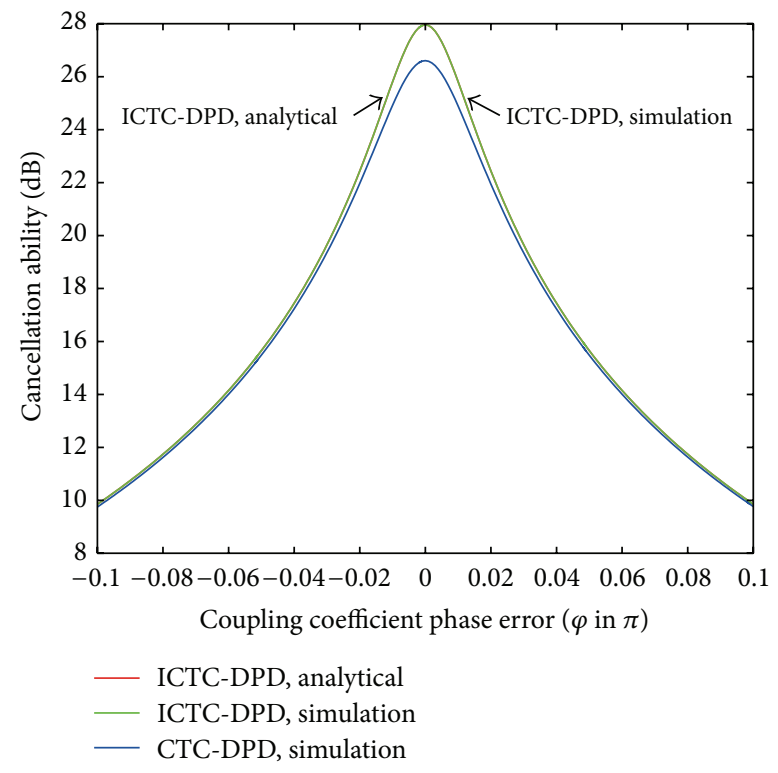

FIGURE 6: Cross talk cancellation ability varies coupling coefficient phase error when magnitude error $\eta=0.04$ in a 2-output MIMO.

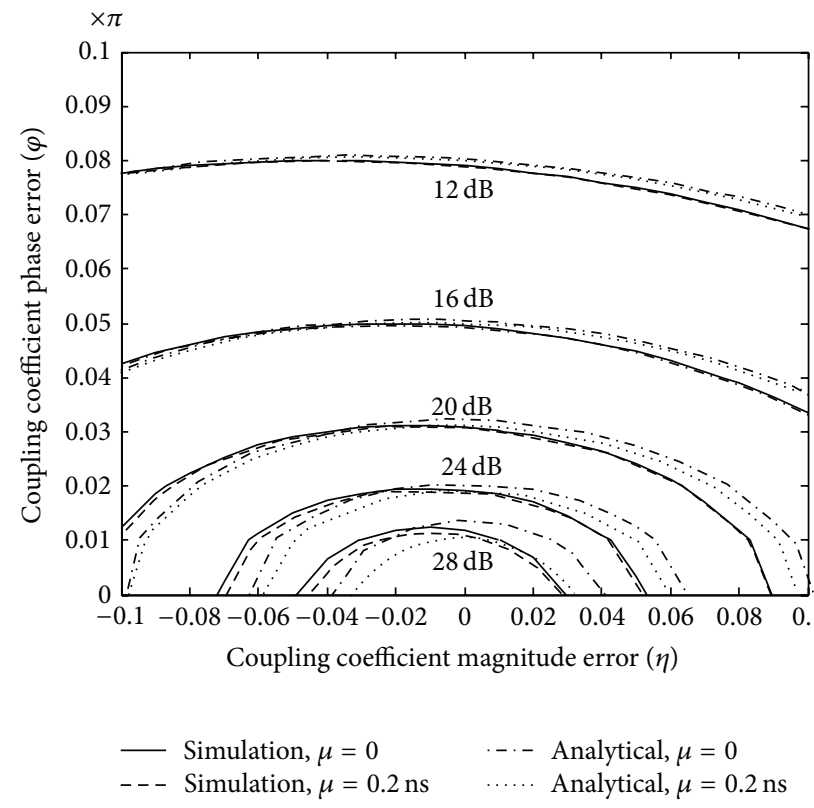

FIGURE 7: Simulation results of cross talk cancellation ability contour for the cross talk between adjacent channels in a 3-output MIMO with ICTC-DPD.

phase error by contour lines, Figure 5 gives cross talk cancellation ability changing with coupling coefficient magnitude error when phase error $\varphi=0.03 \pi$, and Figure 6 gives cross talk cancellation ability changing with coupling coefficient phase error when magnitude error $\eta=0.04 \pi$. In these figures, first, the ICTC-DPD analytical results are in accordance with the simulation results, which proves the correctness of the theoretical analysis. Second, the ICTC-DPD achieves higher cross talk cancellation ability than the CTC-DPD when cross talk delay exists and the cancellation ability improvement by

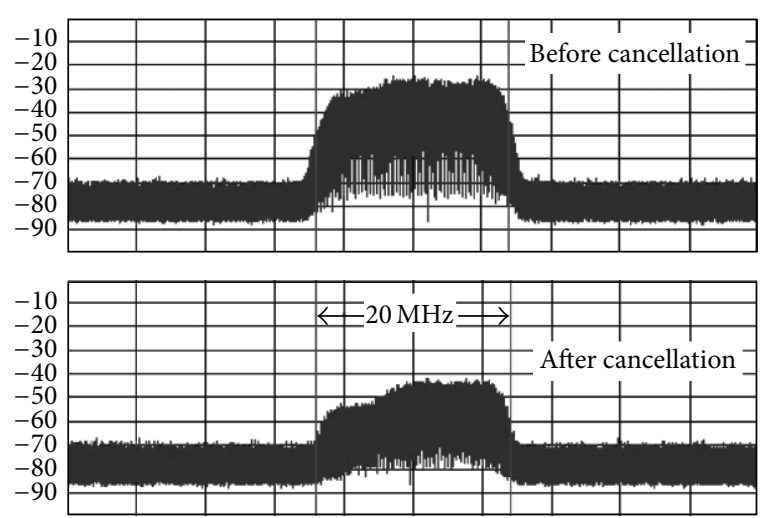

FIGURE 8: Cross talk cancellation for a 2-channel transmitter. Before cancellation, the coupled power in $20 \mathrm{MHz}$ bandwidth is about $-30 \mathrm{dBm}$, and after cancellation it is about $-50 \mathrm{dBm}$, so the cross talk cancellation is about $20 \mathrm{~dB}$.

ICTC-DPD is more significant when the coupling coefficient is more accurate, which prove the effectiveness of ICTCDPD for delayed cross talk. Third, the maximum value and symmetric center of cancellation ability changing with coupling coefficient magnitude error $\eta$ are not at $\eta=0$, which can be revealed by (23).

The results shown in Figure 7 are of a 3-output MIMO with ICTC-DPD compensation. We can see that, first, the contour lines of $\mu=0$ and $\mu=0.2$ ns are almost the same; that is, a cross talk delay estimation error of 0.2 ns leads to insignificant cross talk cancellation ability loss in the ICTCDPD. Second, there is a little difference between the analytical results and the simulation results. This difference arise $s$ because analytical results have ignored cancellation error items introduced by the other channels (from (20) to (21)) when the MIMO has more than 2 outputs.

To test our cross talk cancellation technique, we conduct 2-channel experiments based on the system model shown in Figure 1. The first channel is driven by $20 \mathrm{MHz}$ bandwidth LTE signals with carrier frequency $2.45 \mathrm{GHz}$. The second channel is not driven. The power spectrum of the signals at the coupling port of directional coupler in the second channel is measured.

The power spectrums without and with cross talk cancellation are shown in Figure 8. Before cancellation, the coupled power in $20 \mathrm{MHz}$ bandwidth is about $-30 \mathrm{dBm}$, and after cancellation it is about $-50 \mathrm{dBm}$, so the cross talk cancellation by our technique is about $20 \mathrm{~dB}$ across the $20 \mathrm{MHz}$ bandwidth.

\section{Conclusion}

In this paper, an improved cross talk cancelling digital predistortion with novel estimating technique and decoupling technique is proposed, which can compensate the delayed cross talk, nonlinearity, memory effect, and inconsistency of MIMO transmitting channels. The cross talk cancellation ability of the improved technique is analyzed and its closedform expression is derived. Simulation results prove that this technique can achieve higher cross talk estimated accuracy 
and higher cross talk cancellation ability than the CTC-DPD. Experimental results show that this technique can achieve $20 \mathrm{~dB}$ cross talk cancellation for two channels transmitting $20 \mathrm{MHz}$ bandwidth LTE signals at $2.45 \mathrm{GHz}$.

\section{Appendix}

Proof of (23). By substituting (19), (21), and (22) into (18), we get

$$
\begin{aligned}
& \Psi_{i j} \\
& \approx-10 \log \left[\frac{E\left\{\left|u_{i}\left(t-\tau_{i j}\right)-(1+\eta) e^{j \varphi} u_{i}\left(t-\tau_{i j}-\mu\right)\right|^{2}\right\}}{E\left\{\left|u_{i}\left(t-\tau_{i j}\right)\right|^{2}\right\}}\right] .
\end{aligned}
$$

The numerator in $\log (\cdot)$ can be simplified as

$$
\begin{aligned}
E\left\{\left|u_{i}\left(t-\tau_{i j}\right)-(1+\eta) e^{j \varphi} u_{i}\left(t-\tau_{i j}-\mu\right)\right|^{2}\right\} \\
=E\left\{\begin{array}{c}
\left|u_{i}\left(t-\tau_{i j}\right)\right|^{2}+(1+\eta)^{2}\left|u_{i}\left(t-\tau_{i j}-\mu\right)\right|^{2} \\
-\left[u_{i}\left(t-\tau_{i j}\right)\right]\left[(1+\eta) e^{j \varphi} u_{i}\left(t-\tau_{i j}-\mu\right)\right]^{*} \\
-\left[(1+\eta) e^{j \varphi} u_{i}\left(t-\tau_{i j}-\mu\right)\right]\left[u_{i}\left(t-\tau_{i j}\right)\right]^{*}
\end{array}\right\} \\
=R_{i}(0)+(1+\eta)^{2} R_{i}(0)-(1+\eta) e^{-j \varphi} R_{i}(\mu)
\end{aligned}
$$

The denominator in $\log (\cdot)$ can be simplified as

$$
E\left\{\left|u_{i}\left(t-\tau_{i j}\right)\right|^{2}\right\}=R_{i}(0) .
$$

Therefore, by substituting (A.2) and (A.3) into (A.1), (23) can be attained.

\section{Competing Interests}

The authors declare that there are no competing interests regarding the publication of this paper.

\section{Acknowledgments}

This work was supported by the National Natural Science Foundation of China under Grants nos. 61471108 and 61501093 and by the National Major Projects under Grant no. 2014ZX03003001-002.

\section{References}

[1] R. El Chall, F. Nouvel, M. Hélard, and M. Liu, "Performance and complexity evaluation of iterative receiver for coded MIMOOFDM systems," Mobile Information Systems, vol. 2016, Article ID 7642590, 22 pages, 2016.

[2] X. Su and K. Chang, "Diversity and multiplexing technologies by $3 \mathrm{D}$ beams in polarized massive MIMO systems," Mobile Information Systems, vol. 2016, Article ID 2318287, 15 pages, 2016.
[3] B. M. Hochwald and S. Ten Brink, "Achieving near-capacity on a multiple-antenna channel," IEEE Transactions on Communications, vol. 51, no. 3, pp. 389-399, 2003.

[4] I. E. Telatar, "Capacity of multi-antenna Gaussian channels," European Transactions on Telecommunications, vol. 10, no. 6, pp. 585-595, 1999.

[5] G. J. Foschini and M. J. Gans, "On limits of wireless communications in a fading environment when using multiple antennas," Wireless Personal Communications, vol. 6, no. 3, pp. 311-335, 1998.

[6] 3GPP, TS36.211: Evolved Universal Terrestrial Radio Access (EUTRA); Physical Channels and Modulation (Release 12), 2015.

[7] S. A. Bassam, M. Helaoui, and F. M. Ghannouchi, "Crossover digital predistorter for the compensation of crosstalk and nonlinearity in MIMO transmitters," IEEE Transactions on Microwave Theory and Techniques, vol. 57, no. 5, pp. 1119-1128, 2009.

[8] R. Zayani, R. Bouallegue, and D. Roviras, "Crossover neural network predistorter for the compensation of crosstalk and nonlinearity in MIMO OFDM systems," in Proceedings of the IEEE 21st International Symposium on Personal Indoor and Mobile Radio Communications (PIMRC '10), pp. 966-970, Instanbul, Turkey, September 2010.

[9] P. Suryasarman, M. Hoflehner, and A. Springer, "Digital predistortion for multiple antenna transmitters," in Proceedings of the 43rd European Microwave Conference (EuMC '13), pp. 412415, Nuremberg, Germany, October 2013.

[10] M. V. Amiri, M. Helaoui, and F. M. Ghannouchi, "Streamlined MIMO cross-over digital predistortion," in Proceedings of the IEEE Radio and Wireless Symposium (RWS '14), pp. 283-285, IEEE, Newport Beach, Calif, USA, January 2014.

[11] P. M. Suryasarman and A. Springer, "A comparative analysis of adaptive digital predistortion algorithms for multiple antenna transmitters," IEEE Transactions on Circuits and Systems I: Regular Papers, vol. 62, no. 5, pp. 1412-1420, 2015.

[12] S. Choi and E.-R. Jeong, "Digital predistortion based on combined feedback in MIMO transmitters," IEEE Communications Letters, vol. 16, no. 10, pp. 1572-1575, 2012.

[13] J. Qi and S. Aissa, "Analysis and compensation of power amplifier nonlinearity in MIMO transmit diversity systems," IEEE Transactions on Vehicular Technology, vol. 59, no. 6, pp. 2921-2931, 2010.

[14] H. Lee, B. Lee, and I. Lee, "Iterative detection and decoding with an improved V-BLAST for MIMO-OFDM systems," IEEE Journal on Selected Areas in Communications, vol. 24, no. 3, pp. 504-513, 2006.

[15] D. Tse and P. Viswanath, Fundamentals of Wireless Communication, Cambridge University Press, Cambridge, UK, 2005.

[16] D. R. Morgan, Z. Ma, J. Kim, M. G. Zierdt, and J. Pastalan, “A generalized memory polynomial model for digital predistortion of RF power amplifiers," IEEE Transactions on Signal Processing, vol. 54, no. 10, pp. 3852-3860, 2006.

[17] S. Boyd and L. Vandenberghe, Convex Optimization, Cambridge University Press, Cambridge, UK, 2004. 

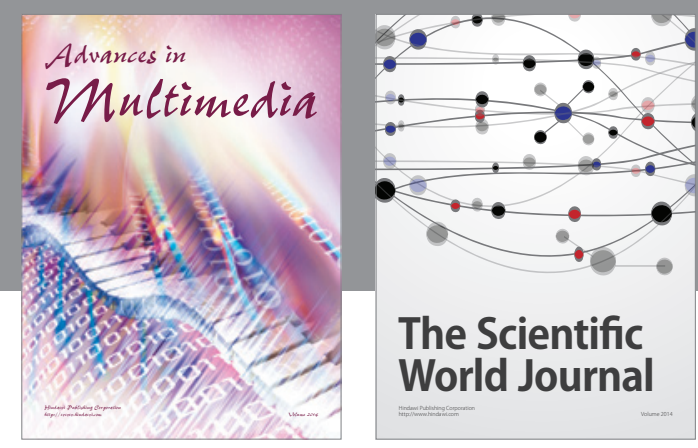

The Scientific World Journal
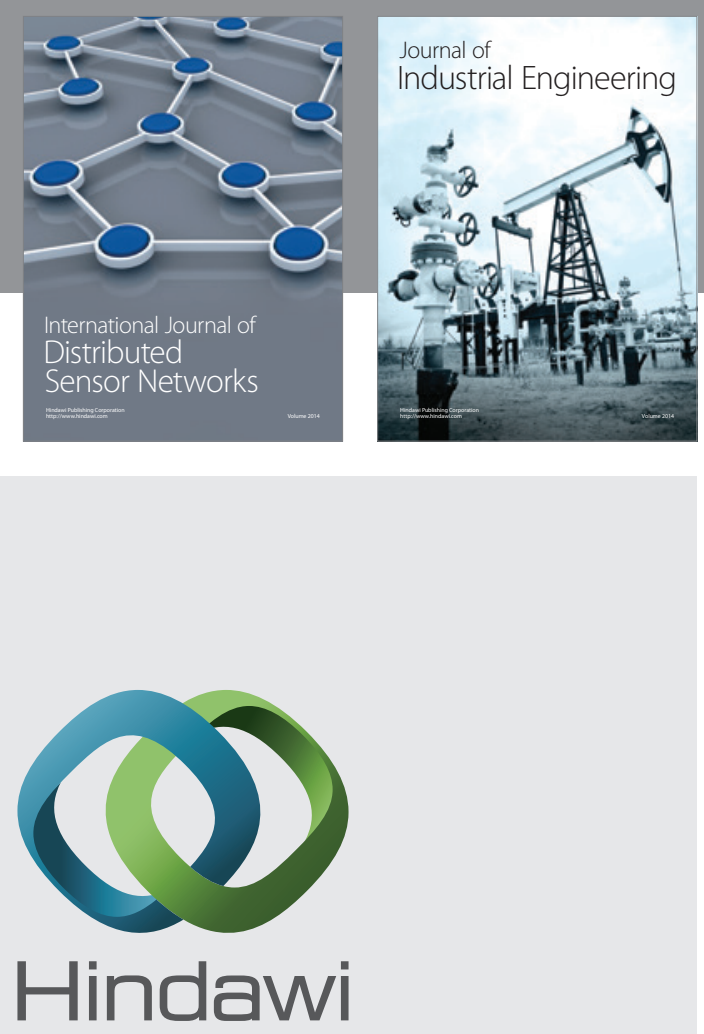

Submit your manuscripts at

http://www.hindawi.com

\section{Computer Networks} and Communications
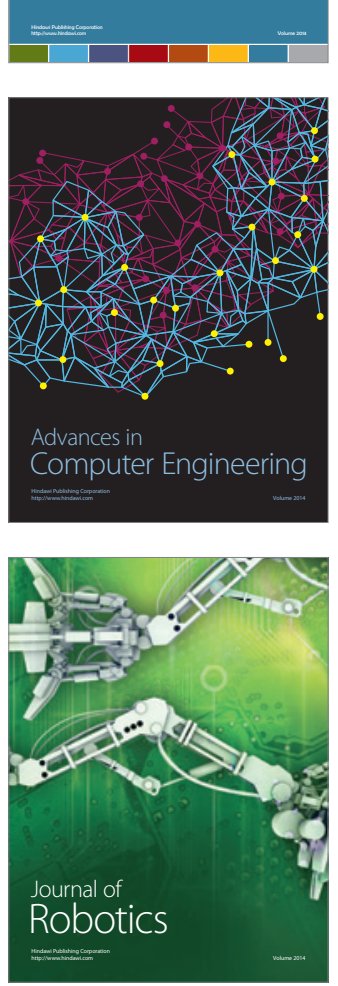
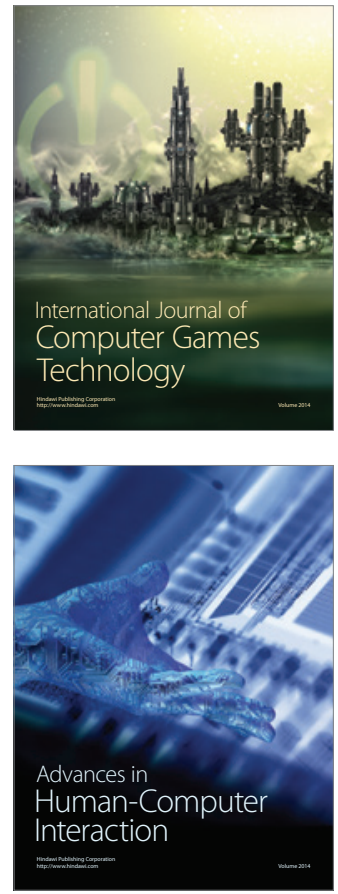
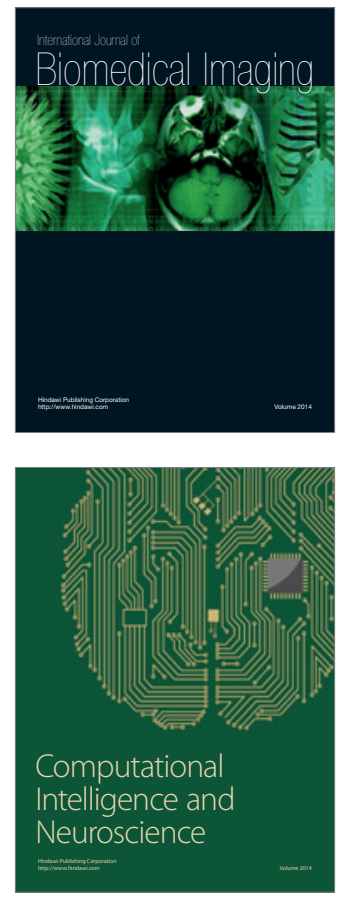
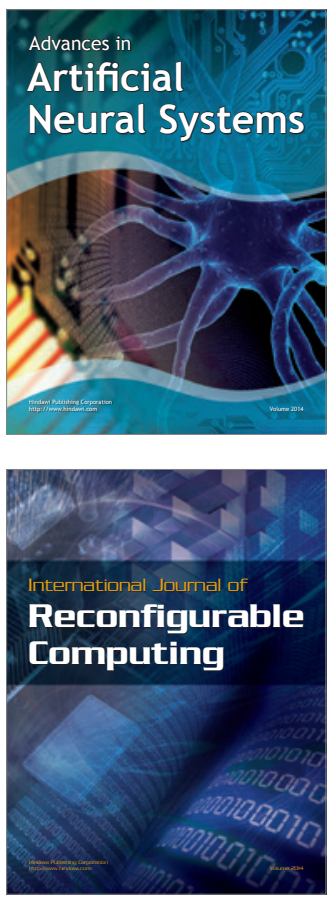
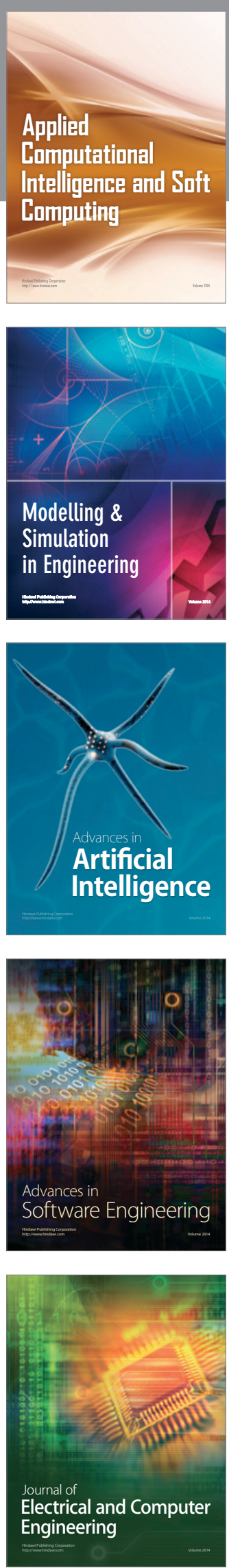